Acta Crystallographica Section E

Structure Reports

Online

ISSN 1600-5368

\section{Picric acid-2,4,6-trichloroaniline (1/1)}

\section{Wan-Qiang Wang}

Department of Chemistry and Biology, Xiangfan University, Xiangfan 441053, People's Republic of China

Correspondence e-mail: wqwang2008@163.com

Received 11 February 2011; accepted 16 February 2011

Key indicators: single-crystal X-ray study; $T=298 \mathrm{~K}$; mean $\sigma(\mathrm{C}-\mathrm{C})=0.005 \AA$; $R$ factor $=0.058 ; w R$ factor $=0.120 ;$ data-to-parameter ratio $=13.1$.

In the title adduct, $\mathrm{C}_{6} \mathrm{H}_{4} \mathrm{Cl}_{3} \mathrm{~N} \cdot \mathrm{C}_{6} \mathrm{H}_{3} \mathrm{~N}_{3} \mathrm{O}_{7}$, the two benzene rings are almost coplanar, with a dihedral angle of $1.19(1)^{\circ}$ and an inter-ring centroid-centroid separation of 4.816 (2) A. The crystal structure is stabilized by intermolecular $\mathrm{N}-$

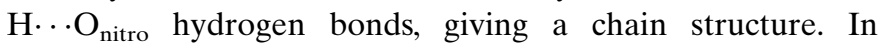
addition, there are phenol-nitro $\mathrm{O}-\mathrm{H} \cdots \mathrm{O}$ interactions.

\section{Related literature}

The crystal structures of picrate salts and picric acid complexes have been studied to investigate charge-transfer processes, see: Nagata et al. (1995); Smith et al. (2004). For the crystal structures of picric acid complexes, see: Li (2009); Sivaramkumar et al. (2010).<smiles>O=[N+]([O-])c1cc([N+](=O)[O-])c(O)c([N+](=O)[O-])c1</smiles><smiles>Nc1c(Cl)cc(Cl)cc1Cl</smiles>

\section{Experimental}

\section{Crystal data}

$\mathrm{C}_{6} \mathrm{H}_{4} \mathrm{Cl}_{3} \mathrm{~N} \cdot \mathrm{C}_{6} \mathrm{H}_{3} \mathrm{~N}_{3} \mathrm{O}_{7}$

$M_{r}=425.57$

Orthorhombic, $\mathrm{Pbca}$

$a=9.2162(14) \AA$
Mo $K \alpha$ radiation
$\mu=0.61 \mathrm{~mm}^{-1}$

Data collection

Bruker SMART CCD area-detector diffractometer

Absorption correction: multi-scan (SADABS; Sheldrick, 1997)

$T_{\min }=0.908, T_{\max }=0.941$

Refinement

$R\left[F^{2}>2 \sigma\left(F^{2}\right)\right]=0.058$

$w R\left(F^{2}\right)=0.120$

$S=1.10$

3186 reflections

244 parameters

2 restraints

Table 1

Hydrogen-bond geometry $\left(\AA{ }^{\circ}\right)$.

\begin{tabular}{lllll}
\hline$D-\mathrm{H} \cdots A$ & $D-\mathrm{H}$ & $\mathrm{H} \cdots A$ & $D \cdots A$ & $D-\mathrm{H} \cdots A$ \\
\hline $\mathrm{O} 1-\mathrm{H} 1 A \cdots \mathrm{O} 7$ & $0.89(4)$ & $1.76(4)$ & $2.546(4)$ & $145(4)$ \\
$\mathrm{N} 4-\mathrm{H} 4 A \cdots \mathrm{O}^{\mathrm{i}}$ & $0.85(2)$ & $2.39(2)$ & $3.159(4)$ & $150(3)$ \\
$\mathrm{N} 4-\mathrm{H} 4 B \cdots 6^{\mathrm{ii}}$ & $0.84(2)$ & $2.40(2)$ & $3.194(4)$ & $156(4)$ \\
\hline
\end{tabular}

Symmetry codes: (i) $-x+\frac{3}{2}, y-\frac{1}{2}, z$; (ii) $-x+\frac{1}{2}, y-\frac{1}{2}, z$.

Data collection: SMART (Bruker, 2001); cell refinement: SAINT (Bruker, 1999); data reduction: $S A I N T$; program(s) used to solve structure: SHELXS97 (Sheldrick, 2008); program(s) used to refine structure: SHELXL97 (Sheldrick, 2008); molecular graphics: SHELXTL (Sheldrick, 2008); software used to prepare material for publication: SHELXTL.

The author is grateful to Xiangfan University for financial support.

Supplementary data and figures for this paper are available from the IUCr electronic archives (Reference: ZS2097).

\section{References}

Bruker (1999). SAINT. Bruker AXS Inc., Madison, Wisconsin, USA. Bruker (2001). SMART. Bruker AXS Inc., Madison, Wisconsin, USA. Li, Y. (2009). Acta Cryst. E65, o2566.

Nagata, H., In, Y., Doi, M., Ishida, T. \& Wakahara, A. (1995). Acta Cryst. B51, 1051-1058.

Sheldrick, G. M. (1997). SADABS. University of Göttingen, Germany.

Sheldrick, G. M. (2008). Acta Cryst. A64, 112-122.

Sivaramkumar, M. S., Velmurugan, R., Sekar, M., Ramesh, P. \& Ponnuswamy, M. N. (2010). Acta Cryst. E66, o1820.

Smith, G., Wermuth, U. D. \& Healy, P. C. (2004). Acta Cryst. E60, o1800-o1803. 


\section{supporting information}

Acta Cryst. (2011). E67, o860 [doi:10.1107/S160053681100571X]

\section{Picric acid-2,4,6-trichloroaniline (1/1)}

\section{Wan-Qiang Wang}

\section{S1. Comment}

2,4,6-Trinitrophenol (picric acid), was primarily used to manufacture explosives and is also used as an intermediate in dye manufacturing. The crystal structures of a large number of picrate salts and picric acid complexes have been studied to determine the conformational features and to understand charge transfer processes (Li et al., 2009; Nagata et al., 1995; Sivaramkumar et al., 2010, Smith et al., 2004). We herein report the 1:1 cocrystal structure of 2,4,6-trichloroaniline and picric acid $\mathrm{C}_{6} \mathrm{H}_{4} \mathrm{Cl}_{3} \mathrm{~N} . \mathrm{C}_{6} \mathrm{H}_{3} \mathrm{~N}_{3} \mathrm{O}_{7}$ (I) (Fig. 1). In the title adduct, the two phenyl rings are almost coplanar with a dihedral angle of $1.19(1)^{\circ}$ and an inter-ring centroid separation of 4.816 (2) $\AA$. The crystal structure is stabilized by intermolecular $\mathrm{N}-\mathrm{H} \cdots \mathrm{O}_{\text {nitro }}$ hydrogen bonds giving a one-dimensional chain structure and in addition, intramolecular $\mathrm{N}$ $-\mathrm{H} \cdots \mathrm{Cl}$ and phenol $\mathrm{O}-\mathrm{H} \cdots \mathrm{O}($ nitro) interactions are observed (Table 1).

\section{S2. Experimental}

2,4,6-Trichloroaniline $(0.19 \mathrm{~g}, 1.0 \mathrm{mmol})$ and picric acid $(0.23 \mathrm{~g}, 1.0 \mathrm{mmol})$ were dissolved in $\mathrm{MeOH}-\mathrm{CH}_{2} \mathrm{Cl}_{2}(3: 1)$ and the mixture was kept at room temperature for one week. Red crystals suitable for single-crystal X-ray diffraction were obtained.

\section{S3. Refinement}

The $\mathrm{O}-$ and $\mathrm{N}$-bound $\mathrm{H}$ atoms were located in a difference map and refined isotropically. The remaining $\mathrm{H}$ atoms were positioned geometrically $(\mathrm{C}-\mathrm{H}=0.93 \AA)$ and allowed to ride on their parent atoms, with $U_{\text {iso }}(\mathrm{H})=1.2 U_{\text {eq }}(\mathrm{C})$. 


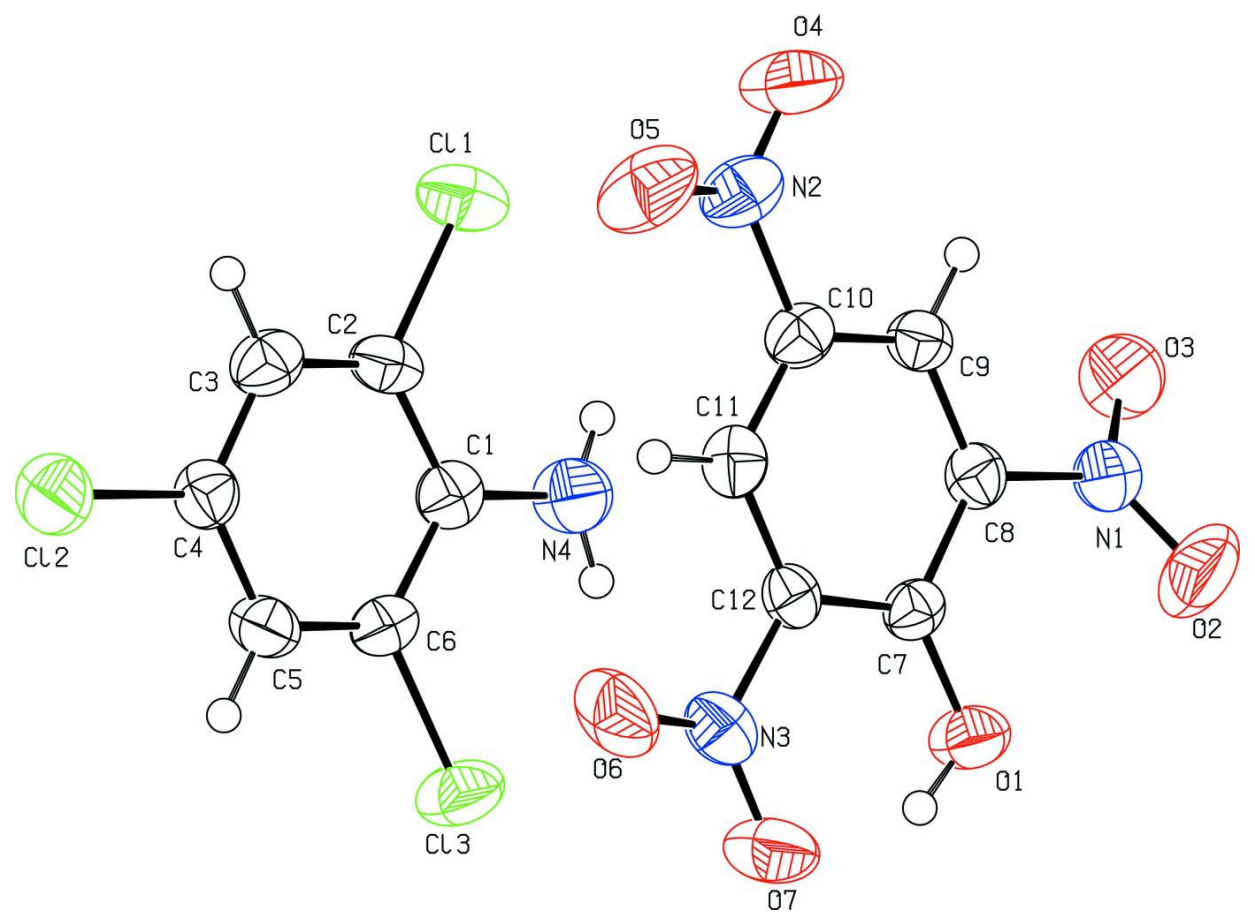

\section{Figure 1}

The title compound with the atom-numbering scheme, with displacement ellipsoids drawn at the $30 \%$ probability level.

\section{Picric acid-2,4,6-trichloroaniline (1/1)}

\section{Crystal data}

\section{$\mathrm{C}_{6} \mathrm{H}_{4} \mathrm{Cl}_{3} \mathrm{~N} \cdot \mathrm{C}_{6} \mathrm{H}_{3} \mathrm{~N}_{3} \mathrm{O}_{7}$}

$M_{r}=425.57$

Orthorhombic, $\mathrm{Pbca}$

Hall symbol: $-\mathrm{P} 2 \mathrm{ac} 2 \mathrm{ab}$

$a=9.2162(14) \AA$

$b=10.0174(14) \AA$

$c=35.051(5) \AA$

$V=3236.0(8) \AA^{3}$

$Z=8$

\section{Data collection}

Bruker SMART CCD area-detector diffractometer

Radiation source: fine-focus sealed tube Graphite monochromator $\varphi$ and $\omega$ scans

Absorption correction: multi-scan

(SADABS; Sheldrick, 1997)

$T_{\min }=0.908, T_{\max }=0.941$
$F(000)=1712$

$D_{\mathrm{x}}=1.747 \mathrm{Mg} \mathrm{m}^{-3}$

Mo $K \alpha$ radiation, $\lambda=0.71073 \AA$

Cell parameters from 1781 reflections

$\theta=2.5-19.2^{\circ}$

$\mu=0.61 \mathrm{~mm}^{-1}$

$T=298 \mathrm{~K}$

Block, red

$0.16 \times 0.12 \times 0.10 \mathrm{~mm}$

19589 measured reflections

3186 independent reflections

2287 reflections with $I>2 \sigma(I)$

$R_{\text {int }}=0.076$

$\theta_{\text {max }}=26.0^{\circ}, \theta_{\min }=2.3^{\circ}$

$h=-11 \rightarrow 11$

$k=-12 \rightarrow 10$

$l=-41 \rightarrow 43$ 


\section{Refinement}

Refinement on $F^{2}$

Least-squares matrix: full

$R\left[F^{2}>2 \sigma\left(F^{2}\right)\right]=0.058$

$w R\left(F^{2}\right)=0.120$

$S=1.10$

3186 reflections

244 parameters

2 restraints

Primary atom site location: structure-invariant direct methods
Secondary atom site location: difference Fourier map

Hydrogen site location: inferred from neighbouring sites

$\mathrm{H}$ atoms treated by a mixture of independent and constrained refinement

$w=1 /\left[\sigma^{2}\left(F_{\mathrm{o}}^{2}\right)+(0.0381 P)^{2}+1.3007 P\right]$ where $P=\left(F_{\mathrm{o}}^{2}+2 F_{\mathrm{c}}^{2}\right) / 3$

$(\Delta / \sigma)_{\max }=0.001$

$\Delta \rho_{\max }=0.21 \mathrm{e} \AA^{-3}$

$\Delta \rho_{\min }=-0.27$ e $\AA^{-3}$

\section{Special details}

Geometry. All e.s.d.'s (except the e.s.d. in the dihedral angle between two 1.s. planes) are estimated using the full covariance matrix. The cell e.s.d.'s are taken into account individually in the estimation of e.s.d.'s in distances, angles and torsion angles; correlations between e.s.d.'s in cell parameters are only used when they are defined by crystal symmetry. An approximate (isotropic) treatment of cell e.s.d.'s is used for estimating e.s.d.'s involving 1.s. planes.

Refinement. Refinement of $F^{2}$ against ALL reflections. The weighted $R$-factor $w R$ and goodness of fit $S$ are based on $F^{2}$, conventional $R$-factors $R$ are based on $F$, with $F$ set to zero for negative $F^{2}$. The threshold expression of $F^{2}>\sigma\left(F^{2}\right)$ is used only for calculating $R$-factors(gt) $e t c$. and is not relevant to the choice of reflections for refinement. $R$-factors based on $F^{2}$ are statistically about twice as large as those based on $F$, and $R$ - factors based on ALL data will be even larger.

Fractional atomic coordinates and isotropic or equivalent isotropic displacement parameters $\left(\AA^{2}\right)$

\begin{tabular}{|c|c|c|c|c|}
\hline & $x$ & $y$ & $z$ & $U_{\text {iso }} * / U_{\text {eq }}$ \\
\hline $\mathrm{C} 1$ & $0.4507(4)$ & $0.4557(3)$ & $0.57639(9)$ & $0.0373(8)$ \\
\hline $\mathrm{C} 2$ & 0.5792 & 0.4955 & $0.55888(10)$ & $0.0396(8)$ \\
\hline $\mathrm{C} 3$ & $0.5881(4)$ & $0.6056(4)$ & $0.53560(10)$ & $0.0422(9)$ \\
\hline $\mathrm{H} 3$ & 0.6760 & 0.6295 & 0.5245 & $0.051 *$ \\
\hline $\mathrm{C} 4$ & $0.4651(4)$ & $0.6801(3)$ & $0.52893(9)$ & $0.0390(8)$ \\
\hline $\mathrm{C} 5$ & $0.3352(4)$ & $0.6456(3)$ & $0.54541(10)$ & $0.0415(9)$ \\
\hline H5 & 0.2523 & 0.6960 & 0.5408 & $0.050 *$ \\
\hline C6 & 0.3293 & 0.5361 & $0.56874(9)$ & $0.0375(8)$ \\
\hline $\mathrm{C} 7$ & $0.4085(3)$ & $0.4501(3)$ & $0.70238(9)$ & $0.0325(7)$ \\
\hline $\mathrm{C} 8$ & $0.5325(3)$ & $0.3724(3)$ & $0.70914(9)$ & $0.0343(8)$ \\
\hline C9 & $0.6640(3)$ & $0.3992(3)$ & $0.69253(9)$ & $0.0360(8)$ \\
\hline H9 & 0.7437 & 0.3447 & 0.6972 & $0.043^{*}$ \\
\hline $\mathrm{C} 10$ & $0.6760(4)$ & $0.5082(3)$ & $0.66889(9)$ & $0.0379(8)$ \\
\hline C11 & $0.5597(4)$ & $0.5899(3)$ & $0.66136(9)$ & $0.0379(8)$ \\
\hline H11 & 0.5694 & 0.6642 & 0.6456 & $0.046^{*}$ \\
\hline $\mathrm{C} 12$ & 0.4278 & $0.5584(3)$ & $0.67784(9)$ & $0.0324(8)$ \\
\hline $\mathrm{Cl1}$ & $0.73498(10)$ & $0.40282(11)$ & $0.56753(3)$ & $0.0607(3)$ \\
\hline $\mathrm{Cl} 2$ & $0.47396(11)$ & $0.81912(11)$ & $0.49911(3)$ & $0.0592(3)$ \\
\hline $\mathrm{Cl3}$ & $0.16542(10)$ & $0.49502(10)$ & $0.59030(3)$ & $0.0560(3)$ \\
\hline N1 & 0.5268 & $0.2597(3)$ & $0.73550(9)$ & $0.0478(8)$ \\
\hline $\mathrm{N} 2$ & $0.8171(3)$ & $0.5390(4)$ & $0.65186(9)$ & $0.0507(8)$ \\
\hline N3 & 0.3064 & 0.6474 & $0.66942(9)$ & $0.0452(8)$ \\
\hline N4 & $0.4439(4)$ & $0.3486(3)$ & $0.60078(10)$ & $0.0520(8)$ \\
\hline $\mathrm{H} 4 \mathrm{~A}$ & $0.512(3)$ & $0.290(3)$ & $0.6009(11)$ & $0.062 *$ \\
\hline
\end{tabular}


supporting information

\begin{tabular}{lllll} 
H4B & $0.359(2)$ & $0.332(4)$ & $0.6079(11)$ & $0.062^{*}$ \\
O1 & $0.2826(3)$ & $0.4170(2)$ & $0.71812(7)$ & $0.0494(7)$ \\
H1A & $0.218(5)$ & $0.479(4)$ & $0.7114(11)$ & $0.074 *$ \\
O2 & $0.4487(4)$ & $0.2669(3)$ & $0.76291(9)$ & $0.0979(13)$ \\
O3 & $0.6055(3)$ & $0.1642(3)$ & $0.72928(8)$ & $0.0643(8)$ \\
O4 & $0.9144(3)$ & $0.4573(3)$ & $0.65587(9)$ & $0.0711(9)$ \\
O5 & $0.8294(3)$ & $0.6445(3)$ & $0.63491(9)$ & $0.0726(9)$ \\
O6 & $0.3275(3)$ & $0.7422(3)$ & $0.64860(8)$ & $0.0672(8)$ \\
O7 & $0.1878(3)$ & $0.6247(3)$ & $0.68450(8)$ & $0.0602(8)$ \\
\hline
\end{tabular}

Atomic displacement parameters $\left(\AA^{2}\right)$

\begin{tabular}{lllllll}
\hline & $U^{11}$ & $U^{22}$ & $U^{33}$ & $U^{12}$ & $U^{13}$ & $U^{23}$ \\
\hline C1 & $0.041(2)$ & $0.035(2)$ & $0.0352(19)$ & $-0.0034(16)$ & $0.0003(15)$ & $-0.0064(15)$ \\
C2 & $0.0318(19)$ & $0.042(2)$ & $0.045(2)$ & $0.0053(16)$ & $-0.0046(15)$ & $-0.0061(17)$ \\
C3 & $0.0369(19)$ & $0.045(2)$ & $0.045(2)$ & $-0.0084(17)$ & $0.0048(16)$ & $-0.0036(17)$ \\
C4 & $0.0398(19)$ & $0.037(2)$ & $0.040(2)$ & $-0.0048(17)$ & $-0.0010(15)$ & $0.0042(16)$ \\
C5 & $0.0319(18)$ & $0.045(2)$ & $0.047(2)$ & $0.0040(16)$ & $-0.0042(16)$ & $0.0025(17)$ \\
C6 & $0.0333(18)$ & $0.040(2)$ & $0.0395(19)$ & $-0.0019(16)$ & $0.0051(15)$ & $-0.0023(16)$ \\
C7 & $0.0329(18)$ & $0.0345(19)$ & $0.0299(17)$ & $-0.0024(15)$ & $0.0016(14)$ & $-0.0052(14)$ \\
C8 & $0.0383(18)$ & $0.0281(19)$ & $0.0365(18)$ & $0.0010(15)$ & $0.0009(15)$ & $0.0055(14)$ \\
C9 & $0.0330(17)$ & $0.037(2)$ & $0.0377(18)$ & $0.0034(15)$ & $-0.0012(15)$ & $-0.0017(15)$ \\
C10 & $0.0355(19)$ & $0.039(2)$ & $0.0392(19)$ & $-0.0075(16)$ & $0.0032(15)$ & $-0.0038(16)$ \\
C11 & $0.044(2)$ & $0.032(2)$ & $0.0385(19)$ & $-0.0068(16)$ & $-0.0021(15)$ & $0.0007(15)$ \\
C12 & $0.0355(18)$ & $0.0322(19)$ & $0.0296(17)$ & $-0.0002(15)$ & $-0.0040(14)$ & $-0.0033(14)$ \\
C11 & $0.0420(5)$ & $0.0648(7)$ & $0.0754(7)$ & $0.0154(5)$ & $-0.0015(5)$ & $-0.0019(5)$ \\
C12 & $0.0527(6)$ & $0.0609(7)$ & $0.0641(6)$ & $-0.0095(5)$ & $-0.0010(5)$ & $0.0236(5)$ \\
C13 & $0.0402(5)$ & $0.0565(6)$ & $0.0712(7)$ & $-0.0038(5)$ & $0.0176(5)$ & $0.0094(5)$ \\
N1 & $0.0449(18)$ & $0.050(2)$ & $0.0481(19)$ & $0.0053(16)$ & $0.0021(15)$ & $0.0120(15)$ \\
N2 & $0.0446(19)$ & $0.052(2)$ & $0.056(2)$ & $-0.0106(17)$ & $0.0097(16)$ & $-0.0038(17)$ \\
N3 & $0.0457(19)$ & $0.041(2)$ & $0.0492(19)$ & $0.0058(15)$ & $-0.0081(15)$ & $0.0014(15)$ \\
N4 & $0.049(2)$ & $0.045(2)$ & $0.062(2)$ & $0.0031(16)$ & $0.0048(18)$ & $0.0101(17)$ \\
O1 & $0.0370(14)$ & $0.0485(17)$ & $0.0626(17)$ & $0.0027(12)$ & $0.0115(12)$ & $0.0103(13)$ \\
O2 & $0.105(3)$ & $0.114(3)$ & $0.075(2)$ & $0.052(2)$ & $0.051(2)$ & $0.0556(19)$ \\
O3 & $0.0714(19)$ & $0.0479(18)$ & $0.073(2)$ & $0.0191(15)$ & $0.0092(15)$ & $0.0161(14)$ \\
O4 & $0.0390(16)$ & $0.079(2)$ & $0.095(2)$ & $0.0047(16)$ & $0.0162(15)$ & $0.0066(18)$ \\
O5 & $0.070(2)$ & $0.0587(19)$ & $0.089(2)$ & $-0.0161(16)$ & $0.0300(16)$ & $0.0133(17)$ \\
O6 & $0.0654(19)$ & $0.0548(18)$ & $0.081(2)$ & $0.0084(15)$ & $-0.0129(15)$ & $0.0285(16)$ \\
O7 & $0.0393(15)$ & $0.0554(18)$ & $0.086(2)$ & $0.0101(13)$ & $0.0032(14)$ & $0.0066(15)$ \\
& & & & & & \\
\hline & & & & & &
\end{tabular}

Geometric parameters $\left(\AA,{ }^{\circ}\right)$

\begin{tabular}{llll}
\hline $\mathrm{C} 1-\mathrm{N} 4$ & $1.373(4)$ & $\mathrm{C} 9-\mathrm{C} 10$ & $1.374(4)$ \\
$\mathrm{C} 1-\mathrm{C} 2$ & $1.392(5)$ & $\mathrm{C} 9-\mathrm{H} 9$ & 0.9300 \\
$\mathrm{C} 1-\mathrm{C} 6$ & $1.404(5)$ & $\mathrm{C} 10-\mathrm{C} 11$ & $1.375(5)$ \\
$\mathrm{C} 2-\mathrm{C} 3$ & $1.374(5)$ & $\mathrm{C} 10-\mathrm{N} 2$ & $1.464(4)$ \\
$\mathrm{C} 2-\mathrm{C} 11$ & $1.736(3)$ & $\mathrm{C} 11-\mathrm{C} 12$ & $1.382(4)$ \\
$\mathrm{C} 3-\mathrm{C} 4$ & $1.377(5)$ & $\mathrm{C} 11-\mathrm{H} 11$ & 0.9300
\end{tabular}




\begin{tabular}{|c|c|c|c|}
\hline $\mathrm{C} 3-\mathrm{H} 3$ & 0.9300 & $\mathrm{C} 12-\mathrm{N} 3$ & $1.461(4)$ \\
\hline $\mathrm{C} 4-\mathrm{C} 5$ & $1.373(4)$ & $\mathrm{N} 1-\mathrm{O} 2$ & $1.202(4)$ \\
\hline $\mathrm{C} 4-\mathrm{Cl} 2$ & $1.743(3)$ & $\mathrm{N} 1-\mathrm{O} 3$ & $1.221(4)$ \\
\hline $\mathrm{C} 5-\mathrm{C} 6$ & $1.370(4)$ & $\mathrm{N} 2-\mathrm{O} 5$ & $1.218(4)$ \\
\hline $\mathrm{C} 5-\mathrm{H} 5$ & 0.9300 & $\mathrm{~N} 2-\mathrm{O} 4$ & $1.222(4)$ \\
\hline $\mathrm{C} 6-\mathrm{Cl} 3$ & $1.739(3)$ & $\mathrm{N} 3-\mathrm{O} 6$ & $1.213(4)$ \\
\hline $\mathrm{C} 7-\mathrm{O} 1$ & $1.327(4)$ & $\mathrm{N} 3-\mathrm{O} 7$ & $1.235(4)$ \\
\hline $\mathrm{C} 7-\mathrm{C} 12$ & $1.396(4)$ & $\mathrm{N} 4-\mathrm{H} 4 \mathrm{~A}$ & $0.854(18)$ \\
\hline $\mathrm{C} 7-\mathrm{C} 8$ & $1.404(4)$ & $\mathrm{N} 4-\mathrm{H} 4 \mathrm{~B}$ & $0.842(18)$ \\
\hline $\mathrm{C} 8-\mathrm{C} 9$ & $1.371(4)$ & $\mathrm{O} 1-\mathrm{H} 1 \mathrm{~A}$ & $0.89(4)$ \\
\hline $\mathrm{C} 8-\mathrm{N} 1$ & $1.460(4)$ & & \\
\hline $\mathrm{N} 4-\mathrm{C} 1-\mathrm{C} 2$ & $122.5(3)$ & $\mathrm{C} 8-\mathrm{C} 9-\mathrm{H} 9$ & 120.6 \\
\hline $\mathrm{N} 4-\mathrm{C} 1-\mathrm{C} 6$ & $122.0(3)$ & $\mathrm{C} 10-\mathrm{C} 9-\mathrm{H} 9$ & 120.6 \\
\hline $\mathrm{C} 2-\mathrm{C} 1-\mathrm{C} 6$ & $115.4(3)$ & $\mathrm{C} 9-\mathrm{C} 10-\mathrm{C} 11$ & $121.7(3)$ \\
\hline $\mathrm{C} 3-\mathrm{C} 2-\mathrm{C} 1$ & $122.8(3)$ & $\mathrm{C} 9-\mathrm{C} 10-\mathrm{N} 2$ & $119.0(3)$ \\
\hline $\mathrm{C} 3-\mathrm{C} 2-\mathrm{C} 11$ & $118.9(3)$ & $\mathrm{C} 11-\mathrm{C} 10-\mathrm{N} 2$ & $119.3(3)$ \\
\hline $\mathrm{C} 1-\mathrm{C} 2-\mathrm{C} 11$ & $118.3(3)$ & $\mathrm{C} 10-\mathrm{C} 11-\mathrm{C} 12$ & $118.0(3)$ \\
\hline $\mathrm{C} 2-\mathrm{C} 3-\mathrm{C} 4$ & $119.1(3)$ & $\mathrm{C} 10-\mathrm{C} 11-\mathrm{H} 11$ & 121.0 \\
\hline $\mathrm{C} 2-\mathrm{C} 3-\mathrm{H} 3$ & 120.4 & $\mathrm{C} 12-\mathrm{C} 11-\mathrm{H} 11$ & 121.0 \\
\hline $\mathrm{C} 4-\mathrm{C} 3-\mathrm{H} 3$ & 120.4 & $\mathrm{C} 11-\mathrm{C} 12-\mathrm{C} 7$ & $123.2(3)$ \\
\hline $\mathrm{C} 5-\mathrm{C} 4-\mathrm{C} 3$ & $120.7(3)$ & $\mathrm{C} 11-\mathrm{C} 12-\mathrm{N} 3$ & $116.7(3)$ \\
\hline $\mathrm{C} 5-\mathrm{C} 4-\mathrm{Cl} 2$ & $119.6(3)$ & $\mathrm{C} 7-\mathrm{C} 12-\mathrm{N} 3$ & $120.0(3)$ \\
\hline $\mathrm{C} 3-\mathrm{C} 4-\mathrm{Cl} 2$ & $119.8(3)$ & $\mathrm{O} 2-\mathrm{N} 1-\mathrm{O} 3$ & $123.0(3)$ \\
\hline $\mathrm{C} 6-\mathrm{C} 5-\mathrm{C} 4$ & $119.1(3)$ & $\mathrm{O} 2-\mathrm{N} 1-\mathrm{C} 8$ & $118.8(3)$ \\
\hline $\mathrm{C} 6-\mathrm{C} 5-\mathrm{H} 5$ & 120.4 & $\mathrm{O} 3-\mathrm{N} 1-\mathrm{C} 8$ & $118.1(3)$ \\
\hline $\mathrm{C} 4-\mathrm{C} 5-\mathrm{H} 5$ & 120.4 & $\mathrm{O} 5-\mathrm{N} 2-\mathrm{O} 4$ & $124.7(3)$ \\
\hline $\mathrm{C} 5-\mathrm{C} 6-\mathrm{C} 1$ & $122.8(3)$ & $\mathrm{O} 5-\mathrm{N} 2-\mathrm{C} 10$ & $117.7(3)$ \\
\hline $\mathrm{C} 5-\mathrm{C} 6-\mathrm{Cl} 3$ & $118.9(3)$ & $\mathrm{O} 4-\mathrm{N} 2-\mathrm{C} 10$ & $117.6(3)$ \\
\hline $\mathrm{C} 1-\mathrm{C} 6-\mathrm{Cl} 3$ & $118.3(3)$ & $\mathrm{O} 6-\mathrm{N} 3-\mathrm{O} 7$ & $122.9(3)$ \\
\hline $\mathrm{O} 1-\mathrm{C} 7-\mathrm{C} 12$ & $124.2(3)$ & $\mathrm{O} 6-\mathrm{N} 3-\mathrm{C} 12$ & $118.4(3)$ \\
\hline $\mathrm{O} 1-\mathrm{C} 7-\mathrm{C} 8$ & $120.2(3)$ & $\mathrm{O} 7-\mathrm{N} 3-\mathrm{C} 12$ & $118.6(3)$ \\
\hline $\mathrm{C} 12-\mathrm{C} 7-\mathrm{C} 8$ & $115.5(3)$ & $\mathrm{C} 1-\mathrm{N} 4-\mathrm{H} 4 \mathrm{~A}$ & $120(3)$ \\
\hline $\mathrm{C} 9-\mathrm{C} 8-\mathrm{C} 7$ & $122.6(3)$ & $\mathrm{C} 1-\mathrm{N} 4-\mathrm{H} 4 \mathrm{~B}$ & $112(3)$ \\
\hline $\mathrm{C} 9-\mathrm{C} 8-\mathrm{N} 1$ & $116.9(3)$ & $\mathrm{H} 4 \mathrm{~A}-\mathrm{N} 4-\mathrm{H} 4 \mathrm{~B}$ & $123(4)$ \\
\hline $\mathrm{C} 7-\mathrm{C} 8-\mathrm{N} 1$ & $120.4(3)$ & $\mathrm{C} 7-\mathrm{O} 1-\mathrm{H} 1 \mathrm{~A}$ & $108(3)$ \\
\hline $\mathrm{C} 8-\mathrm{C} 9-\mathrm{C} 10$ & $118.9(3)$ & & \\
\hline $\mathrm{N} 4-\mathrm{C} 1-\mathrm{C} 2-\mathrm{C} 3$ & $-177.4(3)$ & $\mathrm{C} 8-\mathrm{C} 9-\mathrm{C} 10-\mathrm{C} 11$ & $-0.4(5)$ \\
\hline $\mathrm{C} 6-\mathrm{C} 1-\mathrm{C} 2-\mathrm{C} 3$ & $-0.1(5)$ & $\mathrm{C} 8-\mathrm{C} 9-\mathrm{C} 10-\mathrm{N} 2$ & $178.5(3)$ \\
\hline $\mathrm{N} 4-\mathrm{C} 1-\mathrm{C} 2-\mathrm{C} 11$ & $1.6(5)$ & $\mathrm{C} 9-\mathrm{C} 10-\mathrm{C} 11-\mathrm{C} 12$ & $-1.0(5)$ \\
\hline $\mathrm{C} 6-\mathrm{C} 1-\mathrm{C} 2-\mathrm{Cl} 1$ & $179.0(2)$ & $\mathrm{N} 2-\mathrm{C} 10-\mathrm{C} 11-\mathrm{C} 12$ & $-180.0(3)$ \\
\hline $\mathrm{C} 1-\mathrm{C} 2-\mathrm{C} 3-\mathrm{C} 4$ & $-0.4(5)$ & $\mathrm{C} 10-\mathrm{C} 11-\mathrm{C} 12-\mathrm{C} 7$ & $1.7(5)$ \\
\hline $\mathrm{C} 11-\mathrm{C} 2-\mathrm{C} 3-\mathrm{C} 4$ & $-179.4(3)$ & $\mathrm{C} 10-\mathrm{C} 11-\mathrm{C} 12-\mathrm{N} 3$ & $179.8(3)$ \\
\hline $\mathrm{C} 2-\mathrm{C} 3-\mathrm{C} 4-\mathrm{C} 5$ & $0.4(5)$ & $\mathrm{O} 1-\mathrm{C} 7-\mathrm{C} 12-\mathrm{C} 11$ & $-179.0(3)$ \\
\hline $\mathrm{C} 2-\mathrm{C} 3-\mathrm{C} 4-\mathrm{Cl} 2$ & $-179.4(3)$ & $\mathrm{C} 8-\mathrm{C} 7-\mathrm{C} 12-\mathrm{C} 11$ & $-0.8(5)$ \\
\hline $\mathrm{C} 3-\mathrm{C} 4-\mathrm{C} 5-\mathrm{C} 6$ & $0.2(5)$ & $\mathrm{O} 1-\mathrm{C} 7-\mathrm{C} 12-\mathrm{N} 3$ & $2.9(5)$ \\
\hline $\mathrm{Cl} 2-\mathrm{C} 4-\mathrm{C} 5-\mathrm{C} 6$ & $179.9(3)$ & $\mathrm{C} 8-\mathrm{C} 7-\mathrm{C} 12-\mathrm{N} 3$ & $-178.8(3)$ \\
\hline
\end{tabular}




$\begin{array}{llll}\mathrm{C} 4-\mathrm{C} 5-\mathrm{C} 6-\mathrm{C} 1 & -0.7(5) & \mathrm{C} 9-\mathrm{C} 8-\mathrm{N} 1-\mathrm{O} 2 & 144.8(4) \\ \mathrm{C} 4-\mathrm{C} 5-\mathrm{C} 6-\mathrm{C} 3 & 178.7(3) & \mathrm{C} 7-\mathrm{C} 8-\mathrm{N} 1-\mathrm{O} 2 & -33.6(5) \\ \mathrm{N} 4-\mathrm{C} 1-\mathrm{C} 6-\mathrm{C} 5 & 178.0(3) & \mathrm{C} 9-\mathrm{C} 8-\mathrm{N} 1-\mathrm{O} 3 & -32.8(5) \\ \mathrm{C} 2-\mathrm{C} 1-\mathrm{C} 6-\mathrm{C} 5 & 0.6(5) & \mathrm{C} 7-\mathrm{C} 8-\mathrm{N} 1-\mathrm{O} 3 & 148.8(3) \\ \mathrm{N} 4-\mathrm{C} 1-\mathrm{C} 6-\mathrm{Cl} 3 & -1.4(5) & \mathrm{C} 9-\mathrm{C} 10-\mathrm{N} 2-\mathrm{O} 5 & -171.2(3) \\ \mathrm{C} 2-\mathrm{C} 1-\mathrm{C} 6-\mathrm{Cl} 3 & -178.8(2) & \mathrm{C} 11-\mathrm{C} 10-\mathrm{N} 2-\mathrm{O} 5 & 7.8(5) \\ \mathrm{O} 1-\mathrm{C} 7-\mathrm{C} 8-\mathrm{C} 9 & 177.6(3) & \mathrm{C} 9-\mathrm{C} 10-\mathrm{N} 2-\mathrm{O} 4 & 8.4(5) \\ \mathrm{C} 12-\mathrm{C} 7-\mathrm{C} 8-\mathrm{C} 9 & -0.7(5) & \mathrm{C} 11-\mathrm{C} 10-\mathrm{N} 2-\mathrm{O} 4 & -172.6(3) \\ \mathrm{O} 1-\mathrm{C} 7-\mathrm{C} 8-\mathrm{N} 1 & -4.1(5) & \mathrm{C} 11-\mathrm{C} 12-\mathrm{N} 3-\mathrm{O} 6 & 0.4(4) \\ \mathrm{C} 12-\mathrm{C} 7-\mathrm{C} 8-\mathrm{N} 1 & 177.6(3) & \mathrm{C} 7-\mathrm{C} 12-\mathrm{N} 3-\mathrm{O} 6 & 178.5(3) \\ \mathrm{C} 7-\mathrm{C} 8-\mathrm{C} 9-\mathrm{C} 10 & 1.4(5) & \mathrm{C} 11-\mathrm{C} 12-\mathrm{N} 3-\mathrm{O} 7 & -178.0(3) \\ \mathrm{N} 1-\mathrm{C} 8-\mathrm{C} 9-\mathrm{C} 10 & -177.0(3) & \mathrm{C} 7-\mathrm{C} 12-\mathrm{N} 3-\mathrm{O} 7 & 0.2(5)\end{array}$

Hydrogen-bond geometry $\left(\AA,{ }^{\circ}\right)$

\begin{tabular}{lllll}
\hline$D-\mathrm{H} \cdots A$ & $D-\mathrm{H}$ & $\mathrm{H} \cdots A$ & $D \cdots A$ & $D-\mathrm{H} \cdots A$ \\
\hline $\mathrm{O} 1-\mathrm{H} 1 A \cdots \mathrm{O} 7$ & $0.89(4)$ & $1.76(4)$ & $2.546(4)$ & $145(4)$ \\
$\mathrm{N} 4-\mathrm{H} 4 A \cdots \mathrm{C} 11$ & $0.85(2)$ & $2.62(4)$ & $2.975(3)$ & $106(3)$ \\
$\mathrm{N} 4-\mathrm{H} 4 A \cdots \mathrm{O} 5^{\mathrm{i}}$ & $0.85(2)$ & $2.39(2)$ & $3.159(4)$ & $150(3)$ \\
$\mathrm{N} 4-\mathrm{H} 4 B \cdots \mathrm{Cl} 3$ & $0.84(2)$ & $2.49(3)$ & $2.979(3)$ & $118(3)$ \\
$\mathrm{N} 4-\mathrm{H} 4 B \cdots \mathrm{O} 66^{\text {ii }}$ & $0.84(2)$ & $2.40(2)$ & $3.194(4)$ & $156(4)$
\end{tabular}

Symmetry codes: (i) $-x+3 / 2, y-1 / 2, z$; (ii) $-x+1 / 2, y-1 / 2, z$. 